\title{
Systemic inflammatory response predicts poor prognoses in Barcelona Clinic Liver Cancer stage B/C hepatocellular carcinoma with transarterial chemoembolization: a prospective study
}

\author{
Zhiliang Huang ${ }^{1,2}$, Weiqun Lu ${ }^{1,2}$, Nanrong Yu ${ }^{1,2}$, Guohua Yang ${ }^{1}$, Houwei Xu ${ }^{1}$, Haiying Liu ${ }^{1}$ \\ ${ }^{1}$ Department of Gastrointestinal Surgical Oncology, Affiliated Cancer Hospital \& Institute of Guangzhou Medical University, Guangzhou 510095, \\ China; ${ }^{2}$ Guangzhou Key Laboratory of "Translational Medicine on Malignant Tumor Treatment”, Guangzhou 510095, China \\ Contributions: (I) Conception and design: Z Huang, H Liu; (II) Administrative support: H Liu; (III) Provision of study materials or patients: W Lu, N \\ Yu, G Yang, H Xu; (IV) Collection and assembly of data: Z Huang, W Lu; (V) Data analysis and interpretation: Z Huang; (VI) Manuscript writing: \\ All authors; (VII) Final approval of manuscript: All authors. \\ Correspondence to: Haiying Liu. Department of Gastrointestinal Surgical Oncology, Affiliated Cancer Hospital \& Institute of Guangzhou Medical \\ University, Guangzhou 510095, China. Email: gzdlhy2018@hotmail.com.
}

Background: Chronic inflammation has been demonstrated to be an important factor in the initiation, promotion, and progression of hepatocellular carcinoma (HCC). The aim of this study was to investigate the prognostic values of systemic inflammation markers in Barcelona Clinic Liver Cancer (BCLC) stage B/C HCC.

Methods: A prospective non-randomized study was performed from June 2016 to May 2017; 51 of 123 BCLC stage B/C HCC patients were enrolled and received transarterial chemoembolization (TACE). Clinical and laboratory data were recorded. Serum IL-6, IL-10, C-reactive protein (CRP), and bloodneutrophil-to-lymphocyte ratio (NLR) levels were analyzed during the perioperative period. Patient prognosis was investigated. Twenty-eight stage A cases and 10 stage B/C patients who received resection were also collected as controls.

Results: Compared to the stage A group, the BCLC stage B/C HCC patients had significantly higher serum IL-6, CRP, and blood NLR levels. Serum IL-6, IL-10, CRP, and blood NLR levels increased significantly 3 days after treatment (TACE/resection) and returned to baseline levels after 30 days. By univariate analyses, tumor size, high pretreatment serum IL-6, CRP, and blood NLR levels predicted worse progression-free survival (PFS) after TACE (log-rank test $\mathrm{P}<0.001, \mathrm{P}=0.007, \mathrm{P}=0.001$, respectively). Multivariate analysis revealed that both high serum IL-6 ( $=0.018)$ and CRP $(\mathrm{P}=0.042)$ were independent predictors of worse PFS, meanwhile blood NLR was the only inflammatory factor associated to overall survival (OS) $(\mathrm{P}=0.046)$.

Conclusions: Serum IL-6, CRP, and blood NLR levels were significantly elevated in stage B/CHCC. Serum IL-6 and CRP were independent predictors of poor PFS while NLR independently predicted worse OS in BCLC stage B/C HCC.

Keywords: Hepatocellular carcinoma (HCC); transarterial chemoembolization (TACE); inflammation; prognoses

Submitted Jan 01, 2019. Accepted for publication Oct 09, 2019.

doi: $10.21037 /$ tcr.2019.10.18

View this article at: http://dx.doi.org/10.21037/tcr.2019.10.18 


\section{Introduction}

Hepatocellular carcinoma (HCC) is the sixth most common neoplasm and the third most common cause of cancerrelated deaths worldwide (1). The survival of most HCC patients is very poor, with a 3 -year survival rate of less than $30 \%$. Few of these patients are eligible for curative therapy because most of them are diagnosed at the intermediate or advanced stage. HCC is frequently the long-term result of chronic hepatitis viral infection, and chronic inflammation has been demonstrated as an important factor in the initiation, promotion, and progression of cancer. The proinflammatory cytokine activation of $\mathrm{NF}-\kappa \mathrm{B}$ kinase/ $\mathrm{NF}-\kappa \mathrm{B}(\mathrm{IKK} / \mathrm{NF}-\kappa \mathrm{B})$ signaling pathway was reported as the central link between chronic inflammation and tumor promotion/progression (2). In addition, various cytokines are involved in the pathogenesis and progression of HCC, regulating the proliferation, growth, aggressiveness, and apoptosis of malignant cells, including tumor growth factorbeta (TGF- $\beta)$, tumor necrosis factor-alpha (TNF- $\alpha)(3)$, interferon-gamma (IFN- $\gamma$ ), and interleukin (IL) (4).

IL-6 and IL-10 are pleiotropic cytokines that both play important roles in the differentiation/growth of various types of cells, and as proinflammatory cytokines in the host defense mechanism and immune response. Marked increases in IL-6 and IL-10 levels have been detected in pathological conditions such as trauma, inflammation, and neoplasia. There is evidence that shows elevated serum levels of both these cytokines in patients with chronic liver diseases, including alcoholic hepatitis, hepatitis B virus (HBV) infection, steatohepatitis, and HCC. Moreover, high levels of IL-6 and IL-10 were considered as negative prognostic predictors in various tumors, including HCC $(5,6)$. Despite the increasing knowledge base regarding the roles of IL-6 and IL-10 with HCC, prognostic values of these cytokines in different HCC stages and the behavior of serum IL-6 and IL-10 after treatment are still debated.

Also, several systemic inflammatory markers have recently been studied as prognostic factors in different types of cancer, such as serum C-reactive protein (CRP) (7) and blood neutrophil-lymphocyte ratio (NLR) $(8,9)$. High preoperative serum CRP level has been found to predicted the early recurrence and poor survival of resectable HCC and colorectal cancer (10), but CRP levels do indicate nonspecific change following treatment (11). Of further note, several studies showed an elevated NLR level is correlated with poorer prognosis in patients with different types of cancer, including HCC (12).
However, the change of inflammatory and the immune status during treatment in HCC patients is unclear. Therefore, we designed a prospective study to investigate the prognostic significance of serum IL-6, IL-10, CRP, and blood NLR in BCLC stage B/C HCC treated with TACE and the relationship between inflammatory markers and clinicopathological factors.

\section{Methods}

\section{Study design}

From June 2016 to May 2017, a prospective nonrandomized comparative study was performed on consecutive patients with Barcelona Clinic Liver Cancer (BCLC) stage B/C HCC at the Affiliated Cancer Hospital \& Institute of Guangzhou Medical University. Patients were treated with TACE by using an emulsion of lipiodol and chemotherapeutic-agents with gelatin sponge embolization according to clinical guidelines. The study also recruited age- and gender-matched stage A HCC cases into the control group. Written informed consent was obtained from patients before they were enrolled in this study. This study protocol was approved by the Ethics Committee of Affiliated Cancer Hospital \& Institute of Guangzhou Medical University, and it followed the ethical guidelines of the 1975 Declaration of Helsinki.

\section{Inclusion and exclusion criteria}

During this study period, patients with primary HCC at the Affiliated Cancer Hospital \& Institute of Guangzhou Medical University were considered for inclusion into this study. The diagnosis of HCC was based on the diagnostic criteria used by the European Association for the Study of the Liver. HCC was diagnosed by at least two radiologic images showing characteristic features of HCC, or one radiologic image showing characteristic features of HCC with elevated serum alpha-fetoprotein (AFP) $(\geq 400 \mathrm{ng} / \mathrm{mL}$ ), or direct histopathologic evidence. Clinical and laboratory data from selected patients were recorded, including biochemical parameters, hematological parameters and tumor status.

Patients who met the following inclusion criteria were enrolled for this study: (I) age between 18 and 75 years; (II) HCC with no previous treatment; (III) Eastern Cooperative Group performance status 0-1; (IV) normal liver or ChildPugh A liver cirrhosis; (V) etiology of HCC: HCC with 
or without cirrhosis were accepted, and only patients with alcohol abuse or/and chronic HBV infection history, or healthy history, were enrolled, excluding the HCV-related HCC or other causes related HCC. Also, BCLC stage B/C HCC had to fit at least one of the following criteria: (I) multiple tumors and main size larger than $3 \mathrm{~cm}$; (II) the presence of segmental (tumor thrombus in the peripheral portal vein of the second or lower-order branch) or major (tumor thrombus in the first portal branch or main portal vein without complete occlusion) portal vein tumor thrombi (PVTT) on imaging; (III) tumor with regional lymph node metastasis or direct invasion of adjacent organs other than the gallbladder. Stage A HCC was defined as a solitary tumor with Child-Pugh A liver function.

Patients were excluded from the study if they had one or more of the following: (I) avascular or hypovascular tumor, which was defined as a tumor that could not be detected by angiography or did not show early enhancement on dynamic computed tomography (CT); (II) diffuse-type $\mathrm{HCC}$, defined as multiple tumors, none more than $3 \mathrm{~cm}$; (III) evidence of hepatic decompensation including ascites, esophageal or gastric variceal bleeding, or hepatic encephalopathy; (IV) distant metastasis; (V) severe underlying cardiac or renal diseases; (VI) PVTT with complete main portal vein occlusion, and without adequate collateral circulation around the occluded portal vein; (VII) ongoing interferon therapy at the time of serum collection; (VIII) certain autoimmune disorders are known to alter IL-6, IL-10, or CRP levels.

\section{Patients}

From June 2016 to May 2017, 123 patients with BCLC stage B/C stage primary HCC at the Affiliated Cancer Hospital \& Institute of Guangzhou Medical University were diagnosed. A total of 54 patients were excluded because they refused to receive transarterial chemoembolization (TACE) or hepatectomy. Finally, we enrolled 69 patients who were eligible for this study. Fifty-one patients received TACE, and surgical resections were performed in the other 18 patients. We also collected 28 age- and gender-matched stage A HCC cases in the control group.

\section{TACE}

TACE treatment was performed following the techniques and process (13). Visceral angiography was performed to assess the arterial blood supply of the liver tumor after a selective catheter was introduced. The regimen with same chemotherapeutic agents and same dosage were used consistently in this study, regardless of tumor number and size. Hepatic artery infusion chemotherapy was performed by using carboplatin $300 \mathrm{mg}$ (Bristol-Myers Squibb, New York, NY, USA), followed by epirubicin 50 mg (Pharmorubicin, Pfizer, New York, NY, USA) and mitomycin C $8 \mathrm{mg}$ (Zhejiang Hisun Pharmaceutical Co. Ltd., Taizhou, Zhejiang, China) mixed with $5 \mathrm{~mL}$ of lipiodol (Lipiodol Ultra-Fluide; Andre' Guerbet Laboratories, Aulnay-Sous-Bois, France) for chemolipiodolization. Pure lipiodol was injected if the chemolipiodolized artery territory did not show stagnant flow. In some cases in which we could not achieve stasis in a tumor-feeding artery with the maximum amount of iodized oil $(30 \mathrm{~mL})$ because of large tumor, embolization was performed with absorbable gelatin sponge particles (Gelfoam; Hanzhou alc Ltd., Hangzhou, Zhejiang, China) $1-2 \mathrm{~mm}$ in diameter. The injection could be slowed or discontinued if retrograde flow occurred. Patients were observed carefully after treatment, and analgesia was given if necessary.

\section{Hepatic resection procedure}

Surgical resection was performed by our 4 surgeons and one of whom had at least 15 years of experience on hepatic resection. Reductive resection is defined as resection of the main tumor or the main tumor plus visible satellite nodules around the main tumor, with satellite tumors in the remnant liver classified as unresectable, and whatever the resection margin was. In patients with vascular invasion, tumor thrombus in the portal trunk was removed concomitantly with venotomy of the portal vein. The solitary abdominal lymph node metastases were synchronously resected. More than $30 \%$ of the liver remnant was found in every patient who underwent reductive resection. Postoperative adjuvant TACE was started 4 weeks after the initial hepatectomy and repeated every 4-6 weeks until the remnant tumor completely disappeared on angio-CT or disease progressed.

\section{The array of serum $I L-6, I L-10, C R P$, and blood $N L R$}

Venous blood samplings were conducted in the days before treatment and 3 and 30 days after treatment. Blood samples were centrifuged for 10 minutes at $12,000 \mathrm{rpm}$ at $4{ }^{\circ} \mathrm{C}$. Serum was subsequently removed and frozen at $-70^{\circ} \mathrm{C}$ until assayed. IL-6 and IL-10 levels were measured by human Cytometric Bead Array kit (BD, PharMingen) according 
to the manufacturer's instructions using BD Cell Quest software for IL-6 and IL-10 data analysis. Serum CRP levels were analyzed via immunoturbidimetry. Blood NLR was calculated from the differential ratios by dividing the neutrophil ratio and lymphocyte ratio, as part of the routine measurement of each HCC patient.

\section{Follow-up}

This study was closed on September 30th, 2018, and the routine follow-up program was uniform for patients in both the TACE and resection group. Patients had abdominal computed tomography (CT) scans, serum AFP assay, and liver function test during the first month after treatment, and liver CT scans were performed at 3-month intervals during the first post-treatment year. A routine CT scan was performed every 3 months afterward to evaluate the tumor response. Extrahepatic organ examination was also carried out if patients had suspected extrahepatic metastases. To define suspicious lesions demonstrated on CT and/or by elevated AFP, liver magnetic resonance imaging (MRI) was also used. The Response Evaluation Criteria in Solid Tumors (RECIST) was used to measure tumor response. Progression-free survival (PFS) and overall survival (OS) data were recorded.

\section{Statistical analysis}

Continuous variables were described as mean $\pm \mathrm{SD}$ and compared by Student's $t$-test. Categorical data were presented as frequency and analyzed by using Pearson's chi-squared test. The Kaplan-Meier method was used to access actuarial survival. Univariate analysis was performed to access significant differences in clinicopathological characteristics that influence clinical outcome after TACE treatment. Multivariate analysis was performed by Cox regression for significant variables on univariate analysis. All statistical analyses were performed by using the SPSS for Windows version 13.0 (SPSS Inc., Chicago, IL, USA), and statistical significance was taken at the $5 \%$ level.

\section{Results}

\section{Patients}

The study consisted of 46 (90.2\%) men in the TACE group. Compared to stage A HCC, several systemic inflammatory, and biochemical factors were significantly higher in BCLC stage B/C HCC patients, including serum IL-6, CRP, and blood NLR levels. However, serum IL-10 levels were only detectable in 17 stage $\mathrm{B} / \mathrm{C}$ patients and 1 patient of stage A HCC, which showed no significant difference between these two stages. There was no significant difference in demographic and clinical-pathological features between patients in the TACE and resection group. Clinicopathological and demographic data for the study patients are shown in Table 1.

\section{Correlations of serum IL-6, IL-10, CRP, and blood NLR levels with tumor}

Relationships between serum IL-6, IL-10, CRP, blood NLR levels, and clinical-pathological variables in BCLC stage B/C HCC patients were tested. Serum IL-6 and CRP levels were found to associate with several liver injury markers (such as serum AST and ALP). Moreover, positive relations were found between tumor size and the levels of serum IL-6, CRP, and blood NLR. However, no significant correlations were observed between serum IL-10 levels and clinical-pathological variables (Table 2).

\section{Changes of serum IL-6, IL-10, CRP, and blood NLR levels after treatment}

Serum IL-6, IL-10, CRP, and blood NLR levels in 51 BCLC stage $\mathrm{B} / \mathrm{C}$ HCC patients were analyzed before treatment, and 3 and 30 days after treatment. In all treatments that were performed (TACE or resection), serum IL-6, IL-10, CRP, and blood NLR levels were increased significantly in 3 days after treatment and returned to baseline levels after 30 days. Although the levels of serum IL-6, IL-10, CRP, and blood NLR had no significant differences between the days before treatment and 30 days after treatment, all of these markers tended to reduce within a long period after therapy (Table 3).

\section{Serum IL-6, IL-10, CRP, blood NLR levels, and clinical outcome}

This study was censored on September 30th, 2018. Fortyeight $(69.6 \%)$ patients with BCLC stage B/C HCC were diagnosed as tumor progression at a median of 10 [2-20] months since the baseline visit. By univariate analyses, tumor size, high pretreatment serum IL-6, CRP, and blood NLR levels were associated with shorter PFS in the 
Table 1 Comparison of clinico-pathological and demographic features

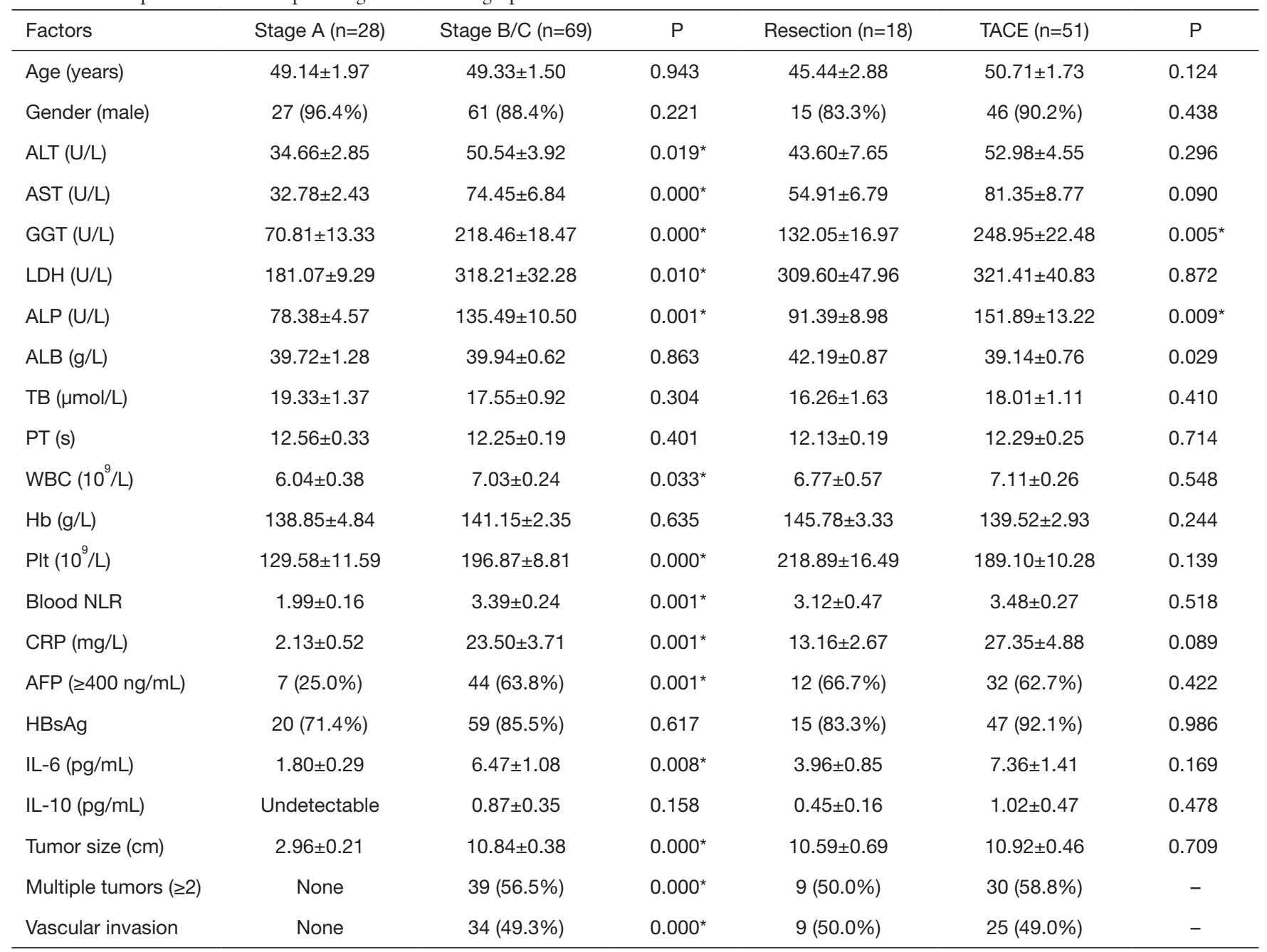

*, $\mathrm{P}<0.05$. TACE, transarterial chemoembolization; ALT, alanine transaminase; AST, aspartate transaminase; GGT, $\gamma$-glutamyl transpeptidase; LDH, L-lactate dehydrogenase; ALP, alkaline phosphatase; ALB, albumin; TB, total bilirubin; PT, prothrombin time; INR, international standard ratio; CRP, C-reactive protein; AFP, alpha-fetoprotein; IL, interleukin; CEA, carcino-embryonic antigen; CA-199, carbohydrate antigen 199; HBsAg, hepatitis B surface antigen; NLR, neutrophil-to-lymphocyte ratio.

BCLC stage B/C HCC patients (Table 4, Figure 1A,B,C). Multivariate analysis revealed that both high serum IL-6 and CRP were independent predictors of PFS.

Moreover, cancer-related death happened in 27 patients during the follow-up period, the 3-month, 6-month, and 9 -month OS rates were $89.6 \%, 70.8 \%$, and $43.8 \%$, respectively. Analysis indicated that blood NLR was the only systemic inflammatory marker, which predicts worse OS in stage B/C HCC with TACE (HR $=2.300,95 \%$ CI: 1.014-5.216, $\mathrm{P}=0.046$, Figure 2). More importantly, compared to pre-treatment level, a lower post-treatment blood NLR level might indicate a better PFS $(11.86 \pm 7.38$ vs. $26.42 \pm 20.13$ weeks, log-rank test $\mathrm{P}=0.028$, Figure $3 A$ ) and OS $(7.14 \pm 4.63$ vs. $11.78 \pm 4.98$ months, log-rank test $\mathrm{P}=0.044$, Figure $3 B$ ) after TACE.

\section{Discussion}

An increasing amount of evidence has indicated that sustained inflammatory reaction playing an important role in HCC development with chronic HBV infection. Various immunocytes and inflammatory cytokines involved in HCC development and progression, including macrophages, neutrophils, $\mathrm{T}$ helper cells, $\mathrm{T}$ regulatory cells, natural 
Table 2 Correlations of serum IL-6, IL-10, CRP and blood NLR levels with clinicopathologic features

\begin{tabular}{|c|c|c|c|c|c|c|c|c|}
\hline Factors & \multicolumn{2}{|c|}{ IL-6, pg/mL } & \multicolumn{2}{|c|}{$\mathrm{IL}-10, \mathrm{pg} / \mathrm{mL}$} & \multicolumn{2}{|c|}{ CRP, mg/L } & \multicolumn{2}{|c|}{ NLR } \\
\hline Age (year) & & 0.670 & & 0.777 & & 0.148 & & 0.133 \\
\hline$\geq 49$ & 6.89 & & 0.96 & & 18.38 & & 3.08 & \\
\hline$<49$ & 5.95 & & 0.76 & & 29.18 & & 3.79 & \\
\hline Male & 7.05 & & 0.97 & & 25.13 & & 3.36 & \\
\hline Female & 2.05 & & 0.14 & & 5.98 & & 3.63 & \\
\hline ALT (U/L) & & 0.095 & & 0.972 & & 0.575 & & 0.556 \\
\hline$\geq 50$ & 8.79 & & 0.89 & & 31.13 & & 3.57 & \\
\hline$\geq 75$ & 10.47 & & 1.16 & & 31.13 & & 4.07 & \\
\hline$<75$ & 4.34 & & 0.72 & & 19.29 & & 3.02 & \\
\hline GGT (U/L) & & 0.006 & & 0.031 & & 0.321 & & 0.060 \\
\hline$\geq 218$ & 10.71 & & 1.97 & & 28.89 & & 4.03 & \\
\hline$<218$ & 4.48 & & 0.36 & & 20.95 & & 3.09 & \\
\hline LDH (U/L) & & 0.070 & & 0.039 & & 0.053 & & 0.038 \\
\hline$\geq 318$ & 10.09 & & 2.13 & & 33.50 & & 4.23 & \\
\hline$<318$ & 5.36 & & 0.36 & & 18.38 & & 3.09 & \\
\hline$<7.0$ & 3.61 & & 0.23 & & 9.99 & & 2.51 & \\
\hline AFP (ng/mL) & & 0.632 & & 0.402 & & 0.444 & & 0.182 \\
\hline$\geq 400$ & 6.85 & & 1.09 & & 25.56 & & 3.63 & \\
\hline$<400$ & 5.75 & & 0.47 & & 19.49 & & 2.97 & \\
\hline $\mathrm{HBsAg}$ & & 0.522 & & 0.542 & & 0.448 & & 0.511 \\
\hline Positive & 6.73 & & 0.94 & & 4.67 & & 3.47 & \\
\hline Negative & 3.70 & & 0 & & 13.29 & & 2.79 & \\
\hline Tumor size & & 0.029 & & 0.065 & & 0.026 & & 0.004 \\
\hline$\geq 10 \mathrm{~cm}$ & 8.40 & & 1.41 & & 30.04 & & 3.95 & \\
\hline$<10 \mathrm{~cm}$ & 3.64 & & 0.09 & & 13.28 & & 2.59 & \\
\hline
\end{tabular}

Table 2 (continued) 
Table 2 (continued)

\begin{tabular}{|c|c|c|c|c|c|c|c|c|}
\hline Factors & \multicolumn{2}{|c|}{ IL-6, pg/mL } & \multicolumn{2}{|c|}{ IL-10, pg/mL } & \multicolumn{2}{|c|}{ CRP, mg/L } & \multicolumn{2}{|c|}{ NLR } \\
\hline Multiple tumors & & 0.076 & & 0.135 & & 0.040 & & 0.845 \\
\hline Yes & 8.47 & & 1.42 & & 31.48 & & 3.34 & \\
\hline No & 4.63 & & 0.37 & & 16.30 & & 3.43 & \\
\hline Yes & 7.83 & & 0.66 & & 25.97 & & 3.73 & \\
\hline No & 5.41 & & 1.09 & & 21.55 & & 3.06 & \\
\hline
\end{tabular}

IL, interleukin; CRP, C-reactive protein; NLR, neutrophil-to-lymphocyte ratio; ALT, alanine transaminase; AST, aspartate transaminase; GGT, $\gamma$-glutamyl transpeptidase; LDH, L-lactate dehydrogenase; ALP, alkaline phosphatase; AFP, alpha-fetoprotein.

Table 3 Levels change of serum IL-6 and IL-10 after treatment

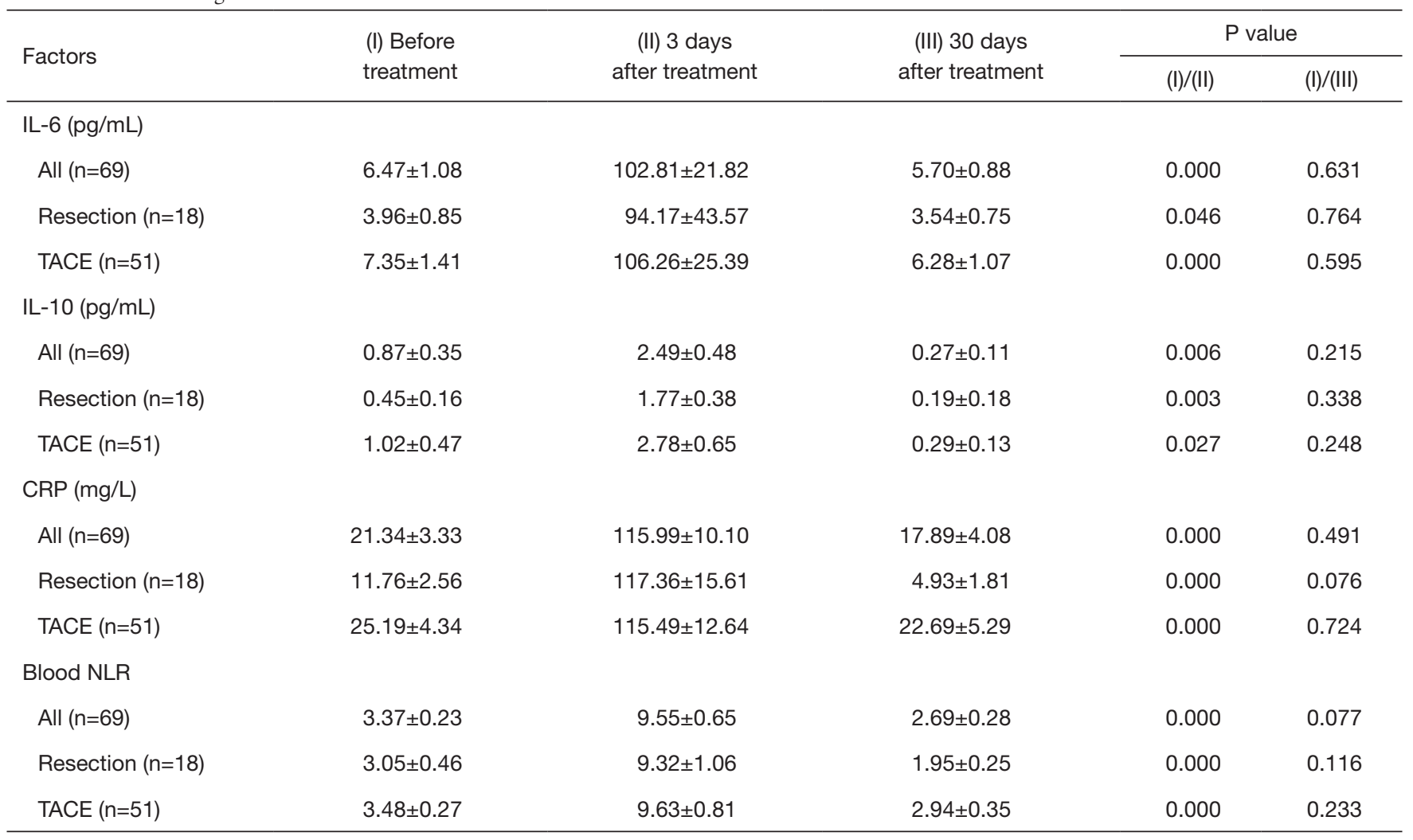

IL, interleukin; CRP, C-reactive protein; TACE, transarterial chemoembolization.

killer cells, and their related cytokines. In this study, we found that serum IL-6, CRP and blood NLR levels were significantly higher in BCLC stage B/C stage HCC patients than in those with early disease, and that these levels were associated with tumor size and liver damage. Importantly, pretreatment serum IL-6 and CRP levels predicted tumor progression, and high NLR level led to worse OS in HCC patients after TACE. Also, compared to stage A cases, these inflammatory markers were found to be significantly elevated in BCLC stage B/C stage HCC. This result suggests tumor-associated inflammation and immune reaction were enhanced during HCC development and 
Table 4 Univariate analyses of clinico-pathological features associated with progression free survival (PFS) in intermediate stage HCC (Spearman's)

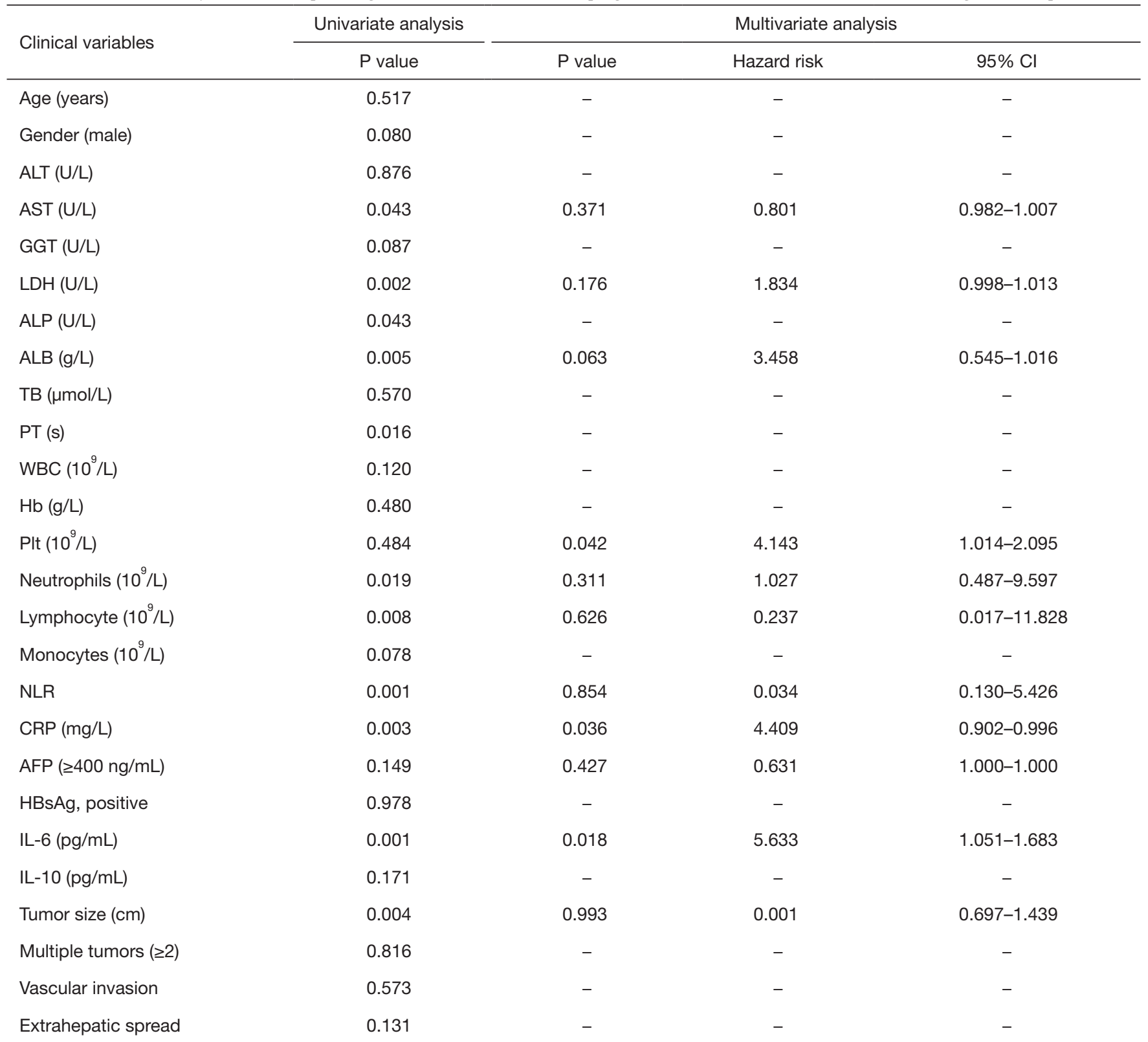

HCC, hepatocellular carcinoma; ALT, alanine transaminase; AST, aspartate transaminase; GGT, $\gamma$-glutamyl transpeptidase; LDH, L-lactate dehydrogenase; ALP, alkaline phosphatase; ALB, albumin; TB, total bilirubin; PT, prothrombin time; NLR, neutrophil-to-lymphocyte ratio; CRP, C-reactive protein; AFP, alpha-fetoprotein; IL, interleukin.

progression, with increasing pro-inflammatory cytokine expression and liver damage.

Cytokines and growth factors produced by tumorinfiltrating macrophages, lymphocytes and other cell types in the inflammatory tumor microenvironment could stimulate cell differentiation and antiapoptosis, which leading to tumor invasiveness and metastasis (4). IL-6 is thought to be one of the most important among various inflammatory cytokines in HCC development.

IL-6 is mainly produced by activated Kupffer cells and up-regulated by HBV X-protein in a mechanism involving nuclear factors $\kappa \mathrm{B}(14,15)$. Although IL-6 is considered to be a hepatoprotective cytokine, it may cause liver damage with high-level expression. Moreover, IL-6 is essential in 
A

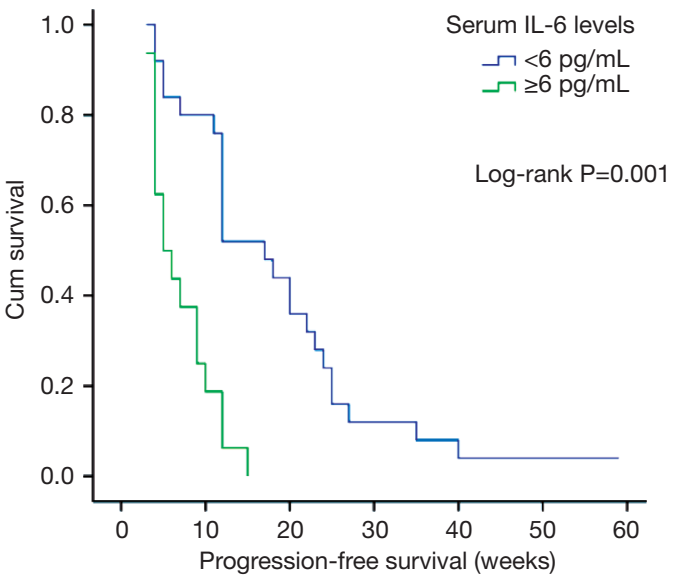

B

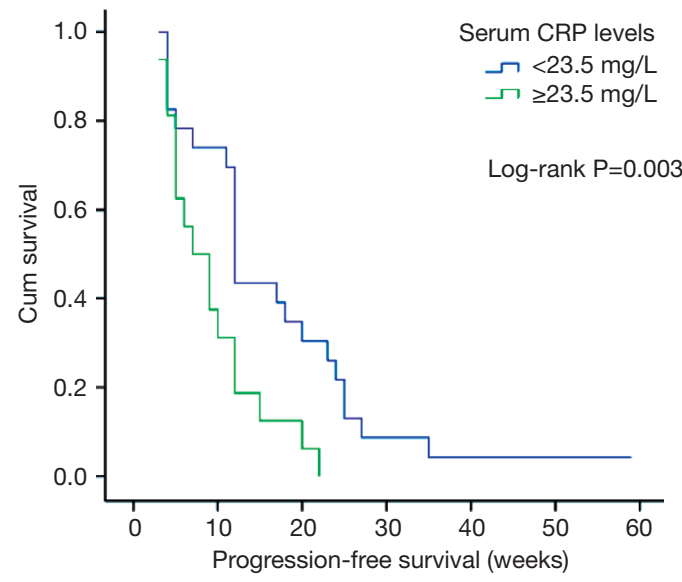

C

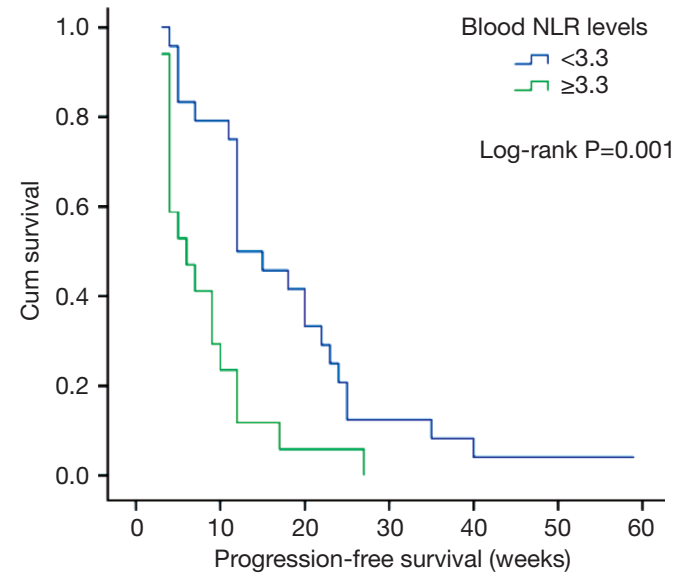

Figure 1 Pretreatment serum IL-6, CRP and blood NLR levels were measured in 51 BCLC stage B/C HCC patients and were divided into high level group and low level group, according to the average of each marker. Log-Rank test showed low IL-6, CRP and NLR groups had better PFS (log-rank test $\mathrm{P}=0.001, \mathrm{P}=0.003$, $\mathrm{P}=0.001$ respectively) than high level groups. CRP, C-reactive protein; NLR, neutrophil-to-lymphocyte ratio; BCLC, Barcelona Clinic Liver Cancer; HCC, hepatocellular carcinoma; PFS, progression-free survival.

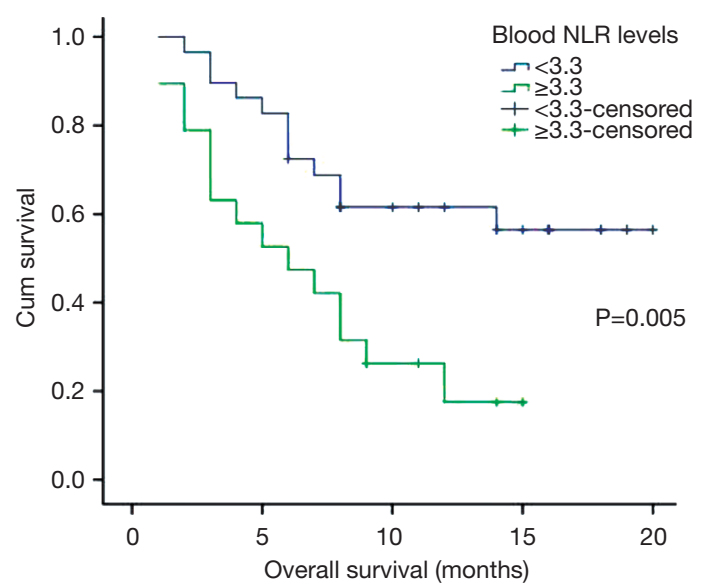

Figure 2 Pretreatment blood NLR were measured in 51 BCLC stage B/C HCC patients with TACE, patients were divided into high level group and low level group, based on the average of NLR. Log-Rank test showed low NLR group had better OS (log-rank test $\mathrm{P}=0.005)$ than high level group. NLR, neutrophilto-lymphocyte ratio; BCLC, Barcelona Clinic Liver Cancer; HCC, hepatocellular carcinoma; TACE, transarterial chemoembolization; OS, overall survival.

the initiation and development of liver cancer likely because of its contribution to promoting hepatocellular damage and compensatory proliferation and carcinogens activation via p-STAT3 (16). Researchers have reported that IL-6 and IL-10 were more elevated in patients with HCC than in those without (5), and were significantly higher in patients with more advanced disease. Higher circulating IL-6, IL-10 levels were considered as useful tumor markers for HCC, especially in a subset of HCC patients with low AFP level $(5,17)$. During HCC development and progression, IL-6 can enhance local inflammatory response and stimulate compensatory hepatocyte proliferation, inducing the malignant transformation of hepatocytes. Animal models studies suggest that high levels of IL-6 can provide the primary stimulus on cell proliferation and be involved in the development of HCC $(4,16)$. IL-6-induced STAT3 signaling in $\mathrm{T}$ cells is critical in promoting Th17 cells differentiation and IL-17A expression, resulting in tumor growth and progression. In contrast, inhibition of IL-6 in macrophage cells may reduce liver cancer in mice $(18,19)$.

With HCC progression, malignancy-related chronic stress and the direct production and secretion by tumorassociated macrophages or tumor cells lead to increase serum IL-6 concentrations (20) and reflect disease status, as serum IL-6 has been found to be significantly higher in 
A

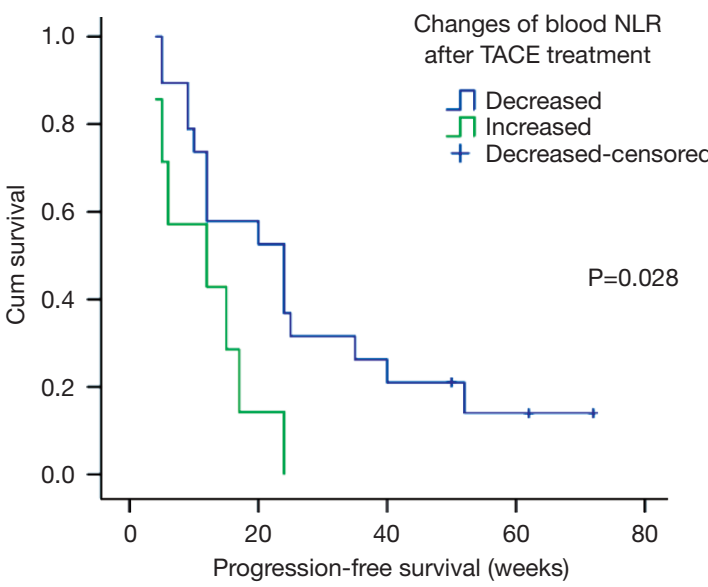

B

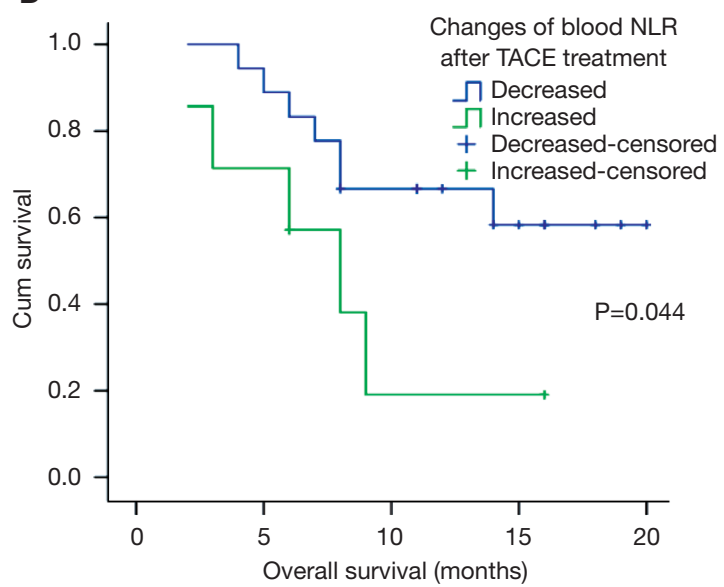

Figure 3 Pre- and post-treatment (30 days) blood NLR levels were measured in 51 BCLC stage B/C HCC patients received TACE, and changes of NLR were analyzed. Patients were divided into NLR increased group and decreased group. Log-Rank test showed NLR decreased group had better PFS (A, P=0.028) and OS (B, P=0.044) than increased group after TACE. NLR, neutrophil-to-lymphocyte ratio; BCLC, Barcelona Clinic Liver Cancer; HCC, hepatocellular carcinoma; TACE, transarterial chemoembolization; PFS, progression-free survival; OS, overall survival.

the advanced stage than in early-stage diseases, and was associated with tumor size in our result. Mounting evidence indicates a prognostic predictor role of IL-6 in different types of solid tumors $(21,22)$.

Besides cytokines, the presence of systemic inflammatory response can be determined by both CRP and blood NLR levels. CRP is synthesized in hepatocytes and produced by other organs in response to the release of IL- 6 by monocytes and immune cells. Because HCC is derived from hepatocytes with a high level of IL-6, it is reasonable to suppose that CRP can be produced by HCC and the induction of IL-6 from tumor cells. Preoperative high serum CRP level has been found to be associated with early recurrence and poor survival in various types of cancer, including HCC $(7,23)$, which is in line with our current results. One study demonstrated that even if curative resection was performed, one-year recurrence rate in HCC patients with high serum CRP levels was $75.3 \%$. This high recurrence rate may be due to the micrometastasis in the liver and peripheral blood before treatment, this cytologic tumor spread cannot be detected either by routine imaging studies or by pathologic examinations, resulting in early recurrence. In addition, some researchers have reported that patients with increased serum CRP had an impaired $\mathrm{T}$ lymphocyte response and a lower infiltration of $\mathrm{CD}^{+} \mathrm{T}$ lymphocytes in the tumor microenvironment. Although this mechanism is still unclear, these findings revealed a possible association between high CRP level and immune system impairment with poor survival.

More and more of the relevant literature has correlated the presence of high blood NLR levels with poor survival in various solid cancers (24), including HCC. High blood NLR may indicate an impaired host immune response to the tumor. A possible explanation is that the host's immune response to the tumor is lymphocyte-dependent, and lymphopenia has been revealed as a predictor of poor survival in several types of cancer. Although the actual mechanisms remain unclear, lymphopenia results from the destruction of lymphocytes elicited by tumor cells or altered homeostasis of lymphocyte pools, a preexisting immune-suppressed condition, or an inadequate induction by cytokines from cancer cells (25). Loss of physiologic homeostasis and subset maintenance could cause a functional impair and ineffective immunologic response of lymphocytes, especially for cytotoxic $\mathrm{T}$ lymphocytes and effector T helper cells. Another possible explanation is that high neutrophil levels can modify and provide an adequate environment for tumor progression and development, by secreting a vast amount of vascular endothelial growth factor (VEGF), angiopoietin-1, and matrix metalloproteinase-9 (MMP-9) $(26,27)$. In addition, these cytokines, which are highly expressed in human cancers, lie at the core of systemic inflammatory and immune responses, including TNF- $\alpha$, interleukin (IL)- $1 \beta$, IL-1R $\alpha$, IL-12, 
and chemokines such as CXCL8 (also known as IL-8), macrophage inflammatory protein (MIP)- $1 \alpha$, and MIP- $1 \beta$ $(28,29)$. Therefore, both lymphocytopenia and high levels of neutrophils have been observed in cancer patients with poor prognosis. A pretreatment elevated blood NLR in patients may suggest a weaker lymphocyte-mediated immune response to tumor and a strong promotion to tumor angiogenesis and antitumor effect suppression, resulting in worsening survival in HCC patients $(30,31)$.

Despite these findings, our current study has some limitations. First, we did not analyze other cytokines which have been reported to be associated with HCC, such as TGF- $\beta$, TNF-a, IFN-r, IL-1, IL-8, IL-12, and IL-17. It is important to describe the entire network between the cytokines response and HCC progression, in addition to their clinical significance. Furthermore, although we discussed the relationship between serum inflammatory markers and HCC progression, the association of $\mathrm{T}$ lymphocyte subsets with tumor progression needs to be further investigated. The relationship between OS and the systemic inflammatory response with a large sample of cases in HCC remains to be examined in a future study.

\section{Conclusions}

In summary, our research revealed pretreatment serum IL-6, CRP, and blood NLR levels were significantly elevated in BCLC stage B/C HCC, and these systemic inflammatory markers may serve as useful predictors of poor PFS and OS in stage B/C HCC patients after TACE. Further study is necessary to evaluate the prognostic value of other cytokines or inflammatory factors in HCC patients and to demonstrate the relationship between systemic inflammatory response and clinical prognosis.

\section{Acknowledgments}

We acknowledge the staff from Intervention Department and Laboratory for technical support.

Funding: None.

\section{Footnote}

Conflicts of Interest: All authors have completed the ICMJE uniform disclosure form (available at http://dx.doi. org/10.21037/tcr.2019.10.18). The authors have no conflicts of interest to declare.
Ethical Statement: The authors are accountable for all aspects of the work in ensuring that questions related to the accuracy or integrity of any part of the work are appropriately investigated and resolved. This study protocol was approved by the Ethics Committee of Affiliated Cancer Hospital \& Institute of Guangzhou Medical University, and it followed the ethical guidelines of the 1975 Declaration of Helsinki (as revised in 2013). Written informed consent was obtained from patients before they were enrolled in this study.

Open Access Statement: This is an Open Access article distributed in accordance with the Creative Commons Attribution-NonCommercial-NoDerivs 4.0 International License (CC BY-NC-ND 4.0), which permits the noncommercial replication and distribution of the article with the strict proviso that no changes or edits are made and the original work is properly cited (including links to both the formal publication through the relevant DOI and the license). See: https://creativecommons.org/licenses/by-nc-nd/4.0/.

\section{References}

1. Forner A, Reig M, Bruix J. Hepatocellular carcinoma. Lancet 2018;391:1301-14.

2. Karin M. Nuclear factor-kappaB in cancer development and progression. Nature 2006;441:431-6.

3. Yamaguchi R, Yano H, Nakashima O, et al. Expression of vascular endothelial growth factor-C in human hepatocellular carcinoma. J Gastroenterol Hepatol 2006;21:152-60.

4. Wan S, Zhao E, Kryczek I, et al. Tumor-associated macrophages produce interleukin 6 and signal via STAT3 to promote expansion of human hepatocellular carcinoma stem cells. Gastroenterology 2014;147:1393-404.

5. Othman MS, Aref AM, Mohamed AA, et al. Serum Levels of Interleukin-6 and Interleukin-10 as Biomarkers for Hepatocellular Carcinoma in Egyptian Patients. ISRN Hepatol 2013;2013:412317.

6. Sheng T, Wand B, Wang SY, et al. The Relationship Between Serum Interleukin-6 and the Recurrence of Hepatitis B Virus Related Hepatocellular Carcinoma after Curative Resection. Medicine (Baltimore) 2015;94:e941.

7. Kinoshita A, Onoda H, Imai N, et al. C-Reactive Protein as a Prognostic Marker in Patients with Hepatocellular Carcinoma. Hepatogastroenterology 2015;62:966-70.

8. Xue TC, Jia QA, Ge NL, et al. Imbalance in systemic inflammation and immune response following transarterial chemoembolization potentially increases metastatic risk in huge hepatocellular carcinoma. Tumour Biol 
2015;36:8797-803.

9. McNally ME, Martinez A, Khabiri H, et al. Inflammatory markers are associated with outcome in patients with unresectable hepatocellular carcinoma undergoing transarterial chemoembolization. Ann Surg Oncol 2013;20:923-8.

10. Morris-Stiff G, Gomez D, Prasad KR. C-reactive protein in liver cancer surgery. Eur J Surg Oncol 2008;34:727-9.

11. Hashimoto K, Ikeda Y, Korenaga D, et al. The impact of preoperative serum C-reactive protein on the prognosis of patients with hepatocellular carcinoma. Cancer 2005;103:1856-64.

12. Huang ZL, Luo J, Chen MS, et al. Blood neutrophilto-lymphocyte ratio predicts survival in patients with unresectable hepatocellular carcinoma undergoing transarterial chemoembolization. J Vasc Interv Radiol 2011;22:702-9.

13. Shi M, Chen JA, Lin XJ, et al. Transarterial chemoembolization as initial treatment for unresectable hepatocellular carcinoma in southern China. World J Gastroenterol 2010;16:264-9.

14. Nakagawa H, Maeda S, Yoshida H, et al. Serum IL-6 levels and the risk for hepatocarcinogenesis in chronic hepatitis $\mathrm{C}$ patients: an analysis based on gender differences. Int J Cancer 2009;125:2264-9.

15. Porta C, De Amici M, Quaglini S, et al. Circulating interleukin-6 as a tumor marker for hepatocellular carcinoma. Ann Oncol 2008;19:353-8.

16. Kao JT, Feng CL, Yu CJ, et al. IL-6, through p-STAT3 rather than p-STAT1, activates hepatocarcinogenesis and affects survival of hepatocellular carcinoma patients: a cohort study. BMC Gastroenterol 2015;15:50.

17. Hsia CY, Huo TI, Chiang SY, et al. Evaluation of interleukin-6, interleukin-10 and human hepatocyte growth factor as tumor markers for hepatocellular carcinoma. Eur J Surg Oncol 2007;33:208-12.

18. Soresi M, Giannitrapani L, D'Antona F, et al. Interleukin-6 and its soluble receptor in patients with liver cirrhosis and hepatocellular carcinoma. World J Gastroenterol 2006; 12:2563-8.

19. Kong L, Zhou Y, Bu H, et al. Deletion of interleukin-6 in monocytes/macrophages suppresses the initiation of hepatocellular carcinoma in mice. J Exp Clin Cancer Res 2016;35:131.

20. Kwon KA, Kim SH, Oh SY, et al. Clinical significance of preoperative serum vascular endothelial growth factor, interleukin-6, and C-reactive protein level in colorectal cancer. BMC Cancer 2010;10:203.

21. Jang JW, Oh BS, Kwon JH, et al. Serum interleukin-6 and C-reactive protein as a prognostic indicator in hepatocellular carcinoma. Cytokine 2012;60:686-93.

22. Hammad LN, Abdelraouf SM, Hassanein FS, et al. Circulating IL-6, IL-17 and vitamin D in hepatocellular carcinoma: potential biomarkers for a more favorable prognosis? J Immunotoxicol 2013;10:380-6.

23. Zhao X, Luo J, Li B, et al. The association between preoperative serum C-reactive protein and hepatocellular carcinoma recurrence in patients with chronic hepatitis $\mathrm{B}$ virus (HBV) infection--a retrospective study. PLoS One 2015;10:e0116909.

24. Sarraf KM, Belcher E, Raevsky E, et al. Neutrophil/ lymphocyte ratio and its association with survival after complete resection in non-small cell lung cancer. J Thorac Cardiovasc Surg 2009;137:425-8.

25. Ray-Coquard I CC, Van Glabbeke M, Sebban C, et al. Lymphopenia as a prognostic factor for overall survival in advanced carcinomas, sarcomas, and lymphomas. Cancer Res 2009;69:5383-91.

26. Behm CZ, Kaufmann BA, Carr C, et al. Molecular imaging of endothelial vascular cell adhesion molecule-1 expression and inflammatory cell recruitment during vasculogenesis and ischemia-mediated arteriogenesis. Circulation 2008;117:2902-11.

27. Gong Y, Koh DR. Neutrophils promote inflammatory angiogenesis via release of preformed VEGF in an in vivo corneal model. Cell Tissue Res 2010;339:437-48.

28. Scapini P, Lapinet-Vera JA, Gasperini S, et al. The neutrophil as a cellular source of chemokines. Immunol Rev 2000;Oct:195-203.

29. Zerbini A, Pilli M, Laccabue D, et al. Radiofrequency thermal ablation for hepatocellular carcinoma stimulates autologous NK-cell response. Gastroenterology 2010;138:1931-42.

30. Aino H, Sumie S, Niizeki T, et al. The systemic inflammatory response as a prognostic factor for advanced hepatocellular carcinoma with extrahepatic metastasis. Mol Clin Oncol 2016;5:83-8.

31. Shi S, Chen Q, Ye L, et al. Prognostic value of systemic inflammation score in patients with hepatocellular carcinoma after hepatectomy. Oncotarget 2017;8:79366-75.

Cite this article as: Huang Z, Lu W, Yu N, Yang G, Xu H, Liu H. Systemic inflammatory response predicts poor prognoses in Barcelona Clinic Liver Cancer stage B/C hepatocellular carcinoma with transarterial chemoembolization: a prospective study. Transl Cancer Res 2019;8(7):2552-2563. doi: 10.21037/ tcr.2019.10.18 